Provided for non-commercial research and education use. Not for reproduction, distribution or commercial use.

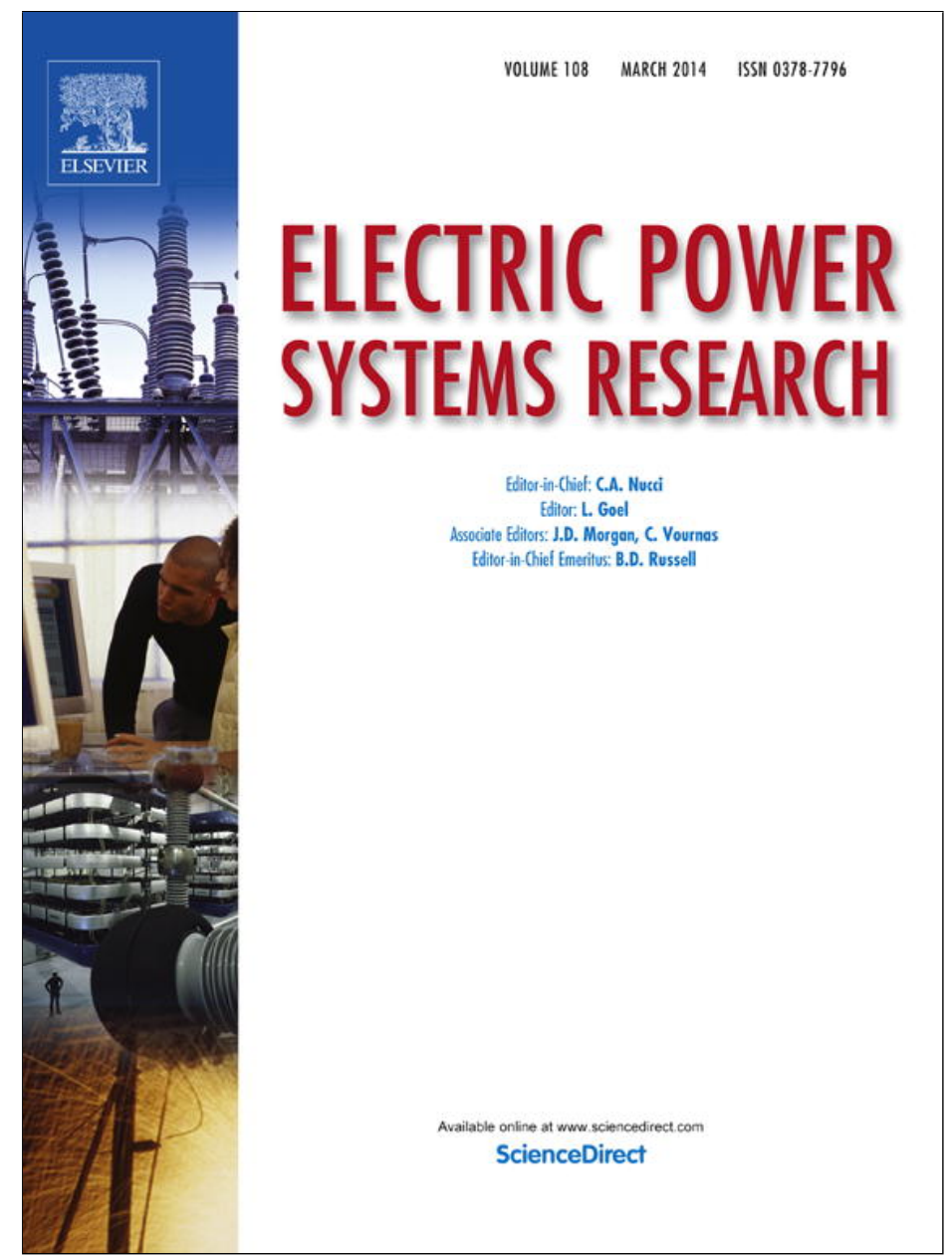

This article appeared in a journal published by Elsevier. The attached copy is furnished to the author for internal non-commercial research and education use, including for instruction at the authors institution and sharing with colleagues.

Other uses, including reproduction and distribution, or selling or licensing copies, or posting to personal, institutional or third party websites are prohibited.

In most cases authors are permitted to post their version of the article (e.g. in Word or Tex form) to their personal website or institutional repository. Authors requiring further information regarding Elsevier's archiving and manuscript policies are encouraged to visit:

http://www.elsevier.com/authorsrights 


\title{
Measurements of harmonic emission versus active power from wind turbines
}

\author{
Kai Yang a,*, Math H.J. Bollen ${ }^{\mathrm{a}, \mathrm{b}}$, E.O. Anders Larsson ${ }^{\mathrm{a}}$, Mats Wahlberg ${ }^{\mathrm{a}, \mathrm{c}}$ \\ a Electric Power Engineering, Luleå University of Technology, 93187 Skellefteå, Sweden \\ b STRI AB, 77180 Ludvika, Sweden \\ c Skellefteå Kraft AB, 93180 Skellefteå, Sweden
}

\section{A R T I C L E I N F O}

\section{Article history:}

Received 10 June 2013

Received in revised form

26 November 2013

Accepted 29 November 2013

Available online 20 December 2013

\section{Keywords:}

Wind energy

Wind power generation

Power quality

Electromagnetic compatibility

Power conversion harmonics

Harmonic analysis

\begin{abstract}
A B S T R A C T
This paper presents harmonic measurements from three individual wind turbines ( 2 and $2.5 \mathrm{MW}$ size). Both harmonics and interharmonics have been evaluated, especially with reference to variations in the active-power production. The overall spectra reveal that emission components may occur at any frequency and not only at odd harmonics. Interharmonics and even harmonics emitted from wind turbines are relatively high. Individual frequency components depend on the power production in different ways: characteristic harmonics are independent of power; interharmonics show a strong correlation with power; other harmonic and interharmonic components present various patterns. It is concluded that the power production is not the only factor determining the current emission of a wind energy conversion system.
\end{abstract}

(c) 2013 Elsevier B.V. All rights reserved.

\section{Introduction}

Modern wind turbines are commonly equipped with power electronics, either with reduced-capacity power converters or fullcapacity power converters. Doubly-fed induction wind generators equipped with reduced-capacity power converters are referred to as type-3, and the wind turbines with full-capacity power converters as type- $4[1,2]$.

The use of power electronics produces waveform distortions [3-7]. A waveform conversion from AC to DC, and then from DC to AC is utilized in the schema of a power converter. The switching of converter involves waveform distortions in the output power $[4,8]$.

The potential consequences of high voltage and current distortion are discussed in various textbooks [9-11]. Waveform distortion is however well regulated in most countries, through compulsory or voluntary limits. The main concern for wind park owners and network operators is to keep distortion levels below those limits.

There are various power conversion schemes part of wind energy conversion systems $[5,8]$. The output waveforms with distortions are determined by the switching schemes. To smooth the

\footnotetext{
* Corresponding author. Tel.: +46 736158980 .

E-mail addresses: kai.yang@ltu.se, epiphany_edwen@hotmail.com (K. Yang).
}

generated harmonics, harmonic filters are used in the wind energy conversion system $[6,8]$. The output distortions are thus dependent on the detailed configuration of the wind turbines.

The standard IEC 61400-21 [12] recommends measurement and assessment of power quality characteristic of grid-connected wind turbines with the measurement method specified in IEC 61000-4-7 [13]. IEC 61400-21 recommends that values of the individual current components (harmonics, interharmonics and higher frequency components) and total harmonic current distortion are measured within the active power bins $0,10,20, \ldots, 100 \%$ of wind turbine rated power. The highest value for each power bin is reported. From a standardization viewpoint there are reasons for selecting one single value, the maximum value in this case. However, to quantify the turbine emission, this is only of use when there are limited variations of the emission within one power bin.

A study based on IEC 61400-21 has been performed on five wind turbines [14]. The current total harmonic distortion is found independent on the output power. Also the fifth harmonic is shown to be independent of the active power for the five wind turbines. The interharmonics and other harmonics have not been presented in reference [14].

A number of $600 \mathrm{~kW}$ squirrel-cage induction generator wind turbines have been tested according to IEC 61400-21 and IEC 61000-4-7 on the medium voltage level [15]. The paper presents an exponentially decreasing of current THD (in percent of fundamental current) with power output; the low order harmonics 3, 5 
and 7 (the dominant ones in THD) are presented without obvious trend with power output.

The aim of this paper is to verify the findings from earlier papers about the relation between active power production and harmonic emission and to extend the study to other harmonics and interharmonics. For this, detailed measurements have been performed on three modern wind turbines, with rating 2 or $2.5 \mathrm{MW}$. Especially the significant levels of interharmonics present in wind power installations, which is much different from conventional installations, has been studied. The risk of the interharmonics and high order harmonics exceeding the limit has been also studied in this paper.

Section 2 describes the measurement setup used to obtain the emission data from the three turbines. Section 3 presents the analysis of wind turbine spectra, whereas Section 4 studies the relation between the emission and the active-power production. And the paper is concluded in Section 5.

\section{Measurement procedure}

\subsection{Measured objects}

Three wind turbines, from different manufacturers and different wind parks, have been measured. All three are 3-blade rotor with horizontal axis, and upwind pitch turbine.

Turbine I: This is a type- 3 wind turbine with a rated power 2.5 MW. It is equipped with a six-pole doubly-fed asynchronous generator (DFIG). A partial rated converter is installed, and designed as a DC voltage link converter with IGBT technology. The rotor outputs a pulse-width modulated (PWM) voltage, while the stator outputs $3 \times 660 \mathrm{~V} / 50 \mathrm{~Hz}$ voltage. The wind turbine is connected to the collection grid through one medium-voltage (MV) transformer, which is housed in a separate transformer station beside the turbine foundation. There are totally 14 such wind turbines within the wind park.

Turbine II: The second wind turbine is also a DFIG type (type-3), whereas the rated power is $2 \mathrm{MW}$. The turbine contains a four-pole doubly-fed asynchronous generator with wound rotor, and a partial rated converter. The output voltage is $690 \mathrm{~V}$, and connected to the collection grid through a medium voltage transformer (installed inside the nacelle). There are 5 such turbines in the park.

Turbine III: The third wind turbine is type-4, with full-power converter. The rated power is $2 \mathrm{MW}$. The turbine is designed based on a gearless drive with synchronous generator. Power is fed into the grid via a full rated converter, a grid side filter and a turbine transformer to the medium voltage level. The inverters are self commutated and pulse the installed IGBTs with variable switching frequency [16].

\subsection{Measurement setup}

Measurements of the above three wind turbines have been performed at the MV side of the turbine transformers (Fig. 1). Both voltage and current have been monitored and recorded using a standard power quality monitor Dranetz-BMI Power Xplorer PX5. Details at the measuring point are listed in Table 1.

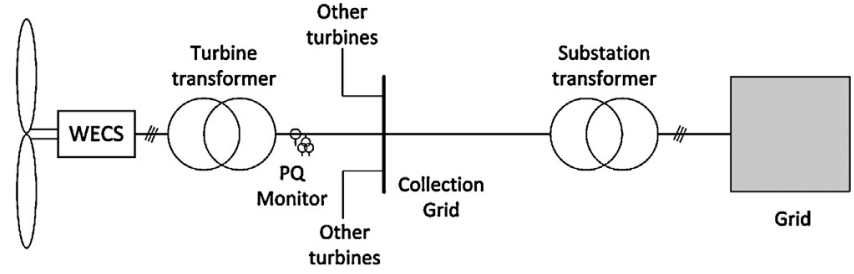

Fig. 1. Power quality monitor position of an individual wind turbine measurement within a wind park.

\subsection{Measurement parameters}

The three-phase voltage and current waveforms were recorded by the monitor through the conventional voltage and current transformers at medium voltage, with a sufficient accuracy for the frequency range up to few $\mathrm{kHz}[17,18]$.

The waveform was continuously acquired with a sampling frequency of 256 times the power-system frequency (approximately $12.8 \mathrm{kHz}$ ) by the power quality monitor. The Discrete Fourier Transform has been applied using a rectangular window of 10 cycles, which results in a $5 \mathrm{~Hz}$ frequency resolution. According to IEC 61000-4-7 [13], the harmonic and interharmonic groups and subgroups are obtained.

Harmonic subgroups $G_{s g, n}$ and interharmonic subgroups $G_{i s g, n}$ at order $n$ are grouped as (for a $50 \mathrm{~Hz}$ system):

$G_{s g, n}{ }^{2}=\sum_{i=-1}^{1} C_{k+1}^{2}$

$G_{i s g, n}{ }^{2}=\sum_{i=2}^{8} C_{k+i}{ }^{2}$

where the harmonic subgroup $G_{s g, n}$ is derived from the 3 Discrete Fourier Transform (DFT) components $C_{k-1}, \ldots, C_{k+1}$ and the interharmonic subgroup $G_{i s g, n}$ is from the 7 DFT components $C_{k+2}, \ldots$, $C_{k+8}$, due to the $5 \mathrm{~Hz}$ resolution spectrum of 10 cycles of sampled waveforms.

The harmonic and interharmonic subgroups, over each 10-cycle window, are next aggregated into 10 -min values. The 10 -min value is the RMS over all 10-cycle values within those $10 \mathrm{~min}$. Details are found in the standard, IEC 61000-4-30.

The grouping and aggregation according to the standard methods take place in the monitor. The user has only access to the grouped and aggregated 10-min values. To allow more detailed analysis, a 10-cycle snapshot of the voltage waveform, with a sample frequency of $12.8 \mathrm{kHz}$, is obtained every $10 \mathrm{~min}$. The analysis presented in this paper is partly based on the aggregated harmonic and interharmonic subgroups and partly on the 200-ms snapshots.

\subsection{Measurement accuracy}

The average spectra, especially for Turbine I, show a broadband spectrum as in Fig. 3 (will be shown later). This spectrum originally raised the suspicion of being due to quantization noise. The spectra

Table 1

Measured objectives details (measure point current and voltage in nominal).

\begin{tabular}{lllcc}
\hline Turbine no. & Generator type & Turbine type & Measure point \\
\cline { 3 - 5 } & & & Current \\
\hline Turbine I & Asynchronous double-fed & Type-3 & $66 \mathrm{~A}$ & Voltage \\
Turbine II & Asynchronous double-fed & Type-3 & $36 \mathrm{~A}$ & $22 \mathrm{kV}$ \\
Turbine III & SYNC-RT & Type-4 & $116 \mathrm{~A}$ & $32 \mathrm{kV}$ \\
\hline
\end{tabular}



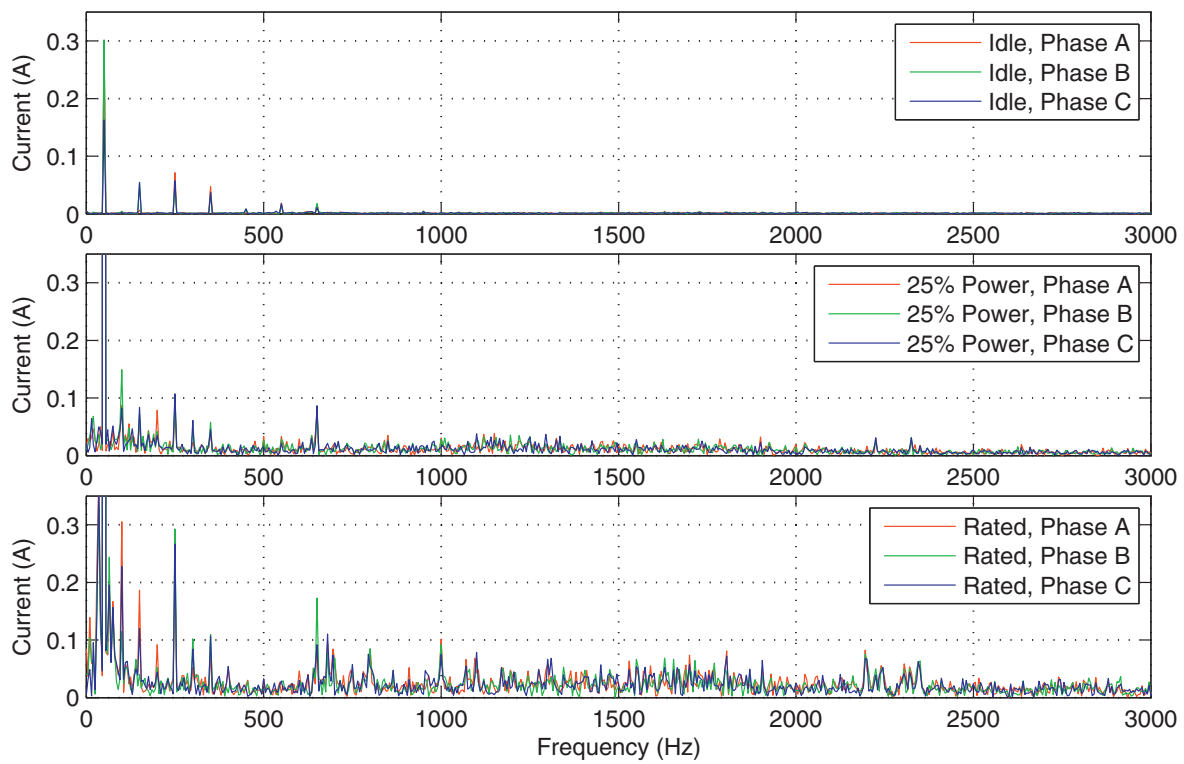

Fig. 2. Spectra of Turbine I at zero, $25 \%$ and rated production.

shown in Fig. 3 have been obtained from the average of a number of spectra obtained from the measurements.

The situation becomes different when looking at individual spectra. A number of such individual spectra for low, medium and high power production are plotted for Turbine I in Fig. 2. The spectra are derived from $200 \mathrm{~ms}$ current waveforms by Discrete Fourier Transform (DFT) at roughly idle, $25 \%$ of rated and rated power production.

The top plot in Fig. 2 presents a spectrum with only a few apparent harmonics at certain narrowbands. The emission is much lower than that of Fig. 3 (will be shown). As the level of quantization noise would be rather independent of the active-power production, it is concluded that the broadband spectrum in Fig. 3 is not due to quantization noise. This conclusion is also confirmed and shown in Fig. 5 (will be shown later), where the blue areas represent low emission, much lower than the level of the broadband. This is the same with the other two turbines.

\section{Wind turbine emission}

\subsection{Emission level}

From the 200-ms windows, spectra with 5-Hz frequency resolution have been obtained. All those spectra, over the measurement period of 8-13 days, have been used to calculate an average spectrum. The average spectra for the three turbines are shown in Fig. 3. Note that this spectrum is obtained from the 10 -cycle snapshots and not from the 10-min grouped values.

The three turbines present a spectrum that is a combination of a broadband spectrum and a number of narrowbands. The narrowbands are mainly centred around integer harmonics, $100 \mathrm{~Hz}, 150 \mathrm{~Hz}, 200 \mathrm{~Hz}$ and $250 \mathrm{~Hz}$. Turbine I (upper sub-figure in Fig. 3) presents a narrowband component centred at $650 \mathrm{~Hz}$ and a broadband component covering neighbouring frequencies. Except the narrowband emission at interger harmonics (e.g. $150 \mathrm{~Hz}$ and

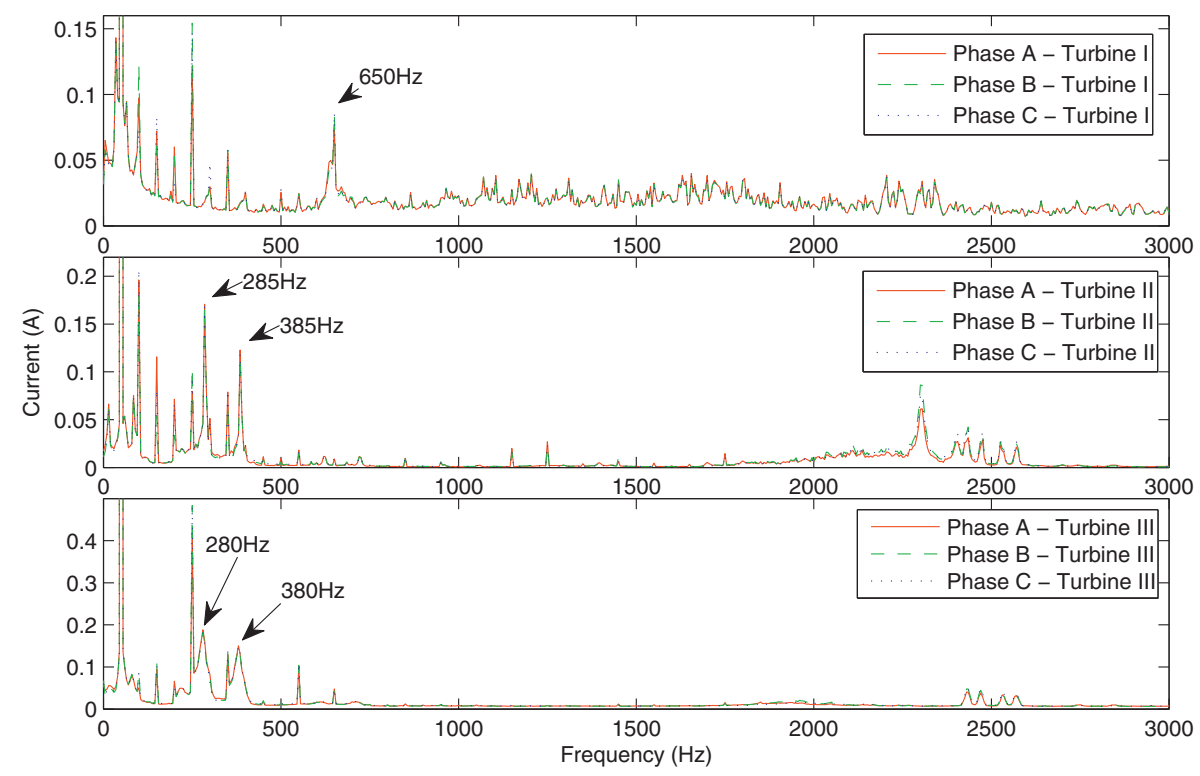

Fig. 3. The average spectra ( $5 \mathrm{~Hz}$ increment) of the three individual wind turbines during a measurement period between 8 and 13 days. 


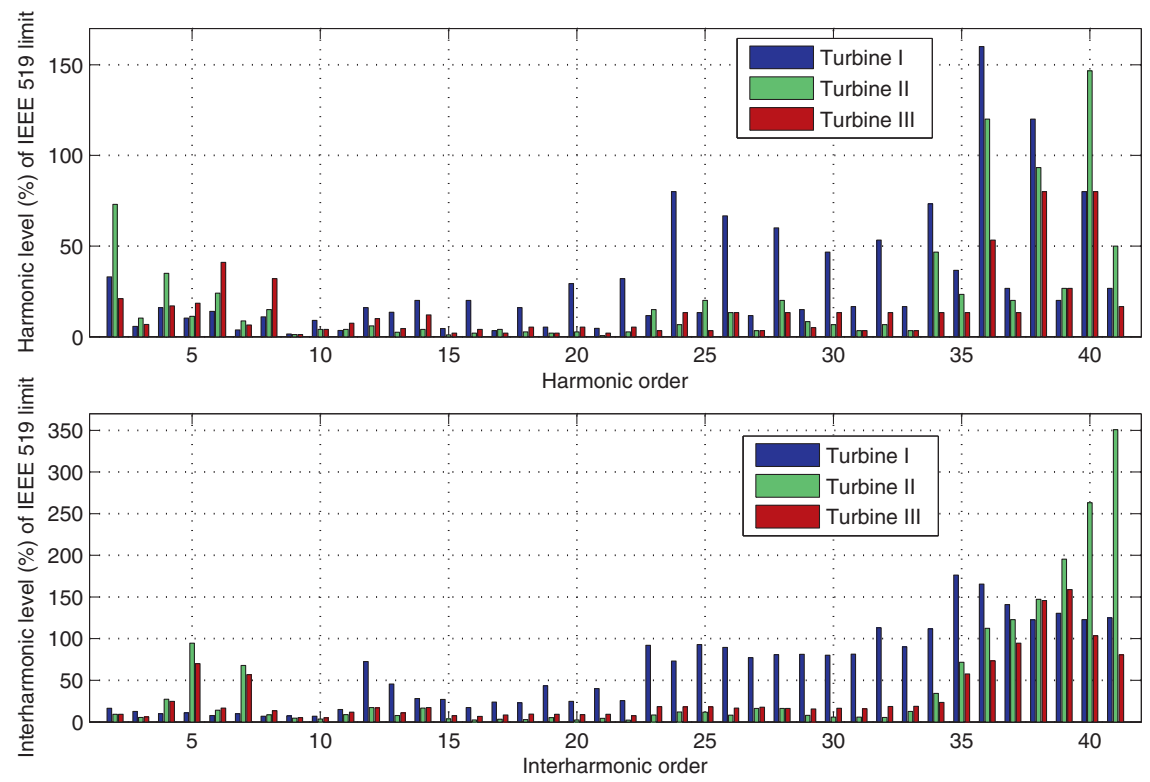

Fig. 4. Upper figure: harmonic level (from harmonic subgroups) as a percentage of the IEEE 519 limits; lower figure: interharmonic level (from interharmonic subgroups) as a percentage of the nearest even harmonic limits of IEEE 519.

$200 \mathrm{~Hz}$ ), Turbine II (middle sub-figure in Fig. 3)) emits obvious narrowband components at $285 \mathrm{~Hz}$ and $385 \mathrm{~Hz}$, and a broadband spectrum up to about $400 \mathrm{~Hz}$ and between 2000 and $2700 \mathrm{~Hz}$. The spectrum of Turbine III (bottom sub-figure) presents two broadband components around $280 \mathrm{~Hz}$ and $380 \mathrm{~Hz}$, next to the narrowband components at harmonics 5 and 7 .

Turbine I presents a broadband component around the fundamental frequency. The side-band components decay with frequency. The centred 5th harmonic $(250 \mathrm{~Hz})$ and broadband around 13th harmonic are apparent. The random and irregular emission between $1 \mathrm{kHz}$ and $2.5 \mathrm{kHz}$ is of interest. The phenomenon is strongly dependent on the configuration of a harmonic filter installed on the turbine side of the turbine transformer.

Turbine II is also DFIG type. The emission is mainly present below $500 \mathrm{~Hz}$ (harmonic 10) and the broadband around $2.3 \mathrm{kHz}$. The components at $285 \mathrm{~Hz}$ and $385 \mathrm{~Hz}$ (close to interharmonic orders 5.5 and 7.5) dominate in the emission. Similarly, Turbine III presents the same dominating emission around the two interharmonic orders. The two dominating components make a large difference from the neighbouring orders.

\subsection{Comparison with limits}

Current emission from the three wind turbines has been compared with the emission limits recommended by IEEE standard 519, which are location independent. The limit for each harmonic order is set in this standard as a percentage of the maximum current. The comparison with IEEE Std.519 is shown in Fig. 4. The vertical scale for both figures is in percent of the IEEE-519 limit for each harmonic or interharmonic. A value above $100 \%$ means that the turbine would not comply with the standard. IEEE Std.519 does not set any limits for interharmonics; for the comparison it was assumed that the interharmonic limits are the same as those for the nearest even harmonic.

The harmonic level is shown as a ratio between the measured harmonic level (harmonic subgroup as a percentage of rated current) and the current harmonic limit for voltage levels through $69 \mathrm{kV}$ as in Fig. 4 (upper figure). The interharmonic level is the ratio between the measured interharmonic level (as a percentage of rated current) and the limits for the nearest even harmonic in IEEE Std.519.
The figure shows that both harmonic and interharmonic levels reach high values at higher orders. It indicates a relatively high emission at high orders at wind turbines, especially the even harmonics. Some even harmonics (order 36, 38 and 40) exceed the $\operatorname{limit}(100 \%)$.

Compared to the harmonic level, interharmonic level shows no obvious difference between neighbouring orders. Also some of the lower-order interharmonics are close to the limit. Higher order interharmonics exceed the limits. The sudden increase at order 24, for both harmonics and interharmonics, is due to lower emission limits for order 24 and higher.

Different from emission of other installations, even harmonics and interharmonics from wind turbines are high compared to the emission limits, especially for higher orders.

\subsection{Primary and secondary emission}

The influence of the background voltage distortion on the current distortion is often discussed informally, but no qualitative results for wind turbines are available from the literature. The standard IEC 61400-21 states that the measurements should be done when the background voltage distortion is low, without further quantifying this. A study for a large off-shore wind park [19] showed that, at the point of connection with the $150-\mathrm{kV}$ grid, the harmonic currents due to background voltage distortion may be several orders of magnitude larger than the currents due to the emission from the turbines.

The impact of background voltage on the current distortion is not limited to wind-power installations. Similar phenomena have been studied for lighting installations at frequencies of some tens of $\mathrm{kHz}$ [20]. In that study a distinction was made between "primary emission" and "secondary emission", which proved very useful when quantifying the interaction between devices.

The primary emission is the distortion generated within the installation itself; while the secondary emission is the distortion generated from somewhere else. What counts as "installation" depends on the location of the point of evaluation. When considering one single turbine, the primary emission is the one due to the turbine itself. The measured emission at a certain turbine is a combination of primary and secondary emission. 


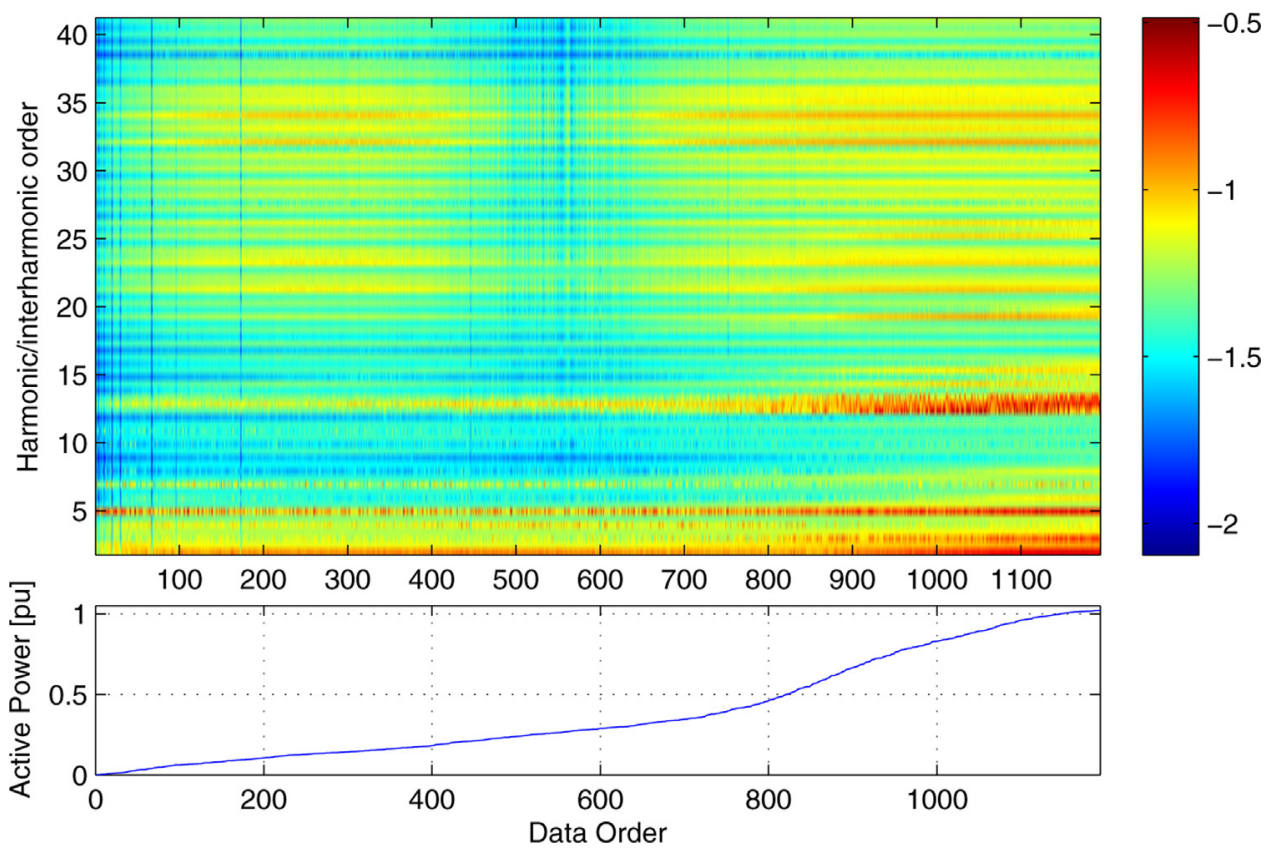

Fig. 5. Turbine I: harmonic ( $\mathrm{H} 2-\mathrm{H} 41)$ and interharmonic ( $\mathrm{IH} 2.5-\mathrm{IH} 41.5)$ subgroups as a function of active power. (For interpretation of the references to colour in the text, the reader is referred to the web version of the article.)

For the turbines studied in this paper, the correlation coefficient has been calculated between the harmonic voltage and harmonic current at a certain harmonic order. Results show that most harmonic orders present a low correlation coefficient with a value around 0.2 . Thus the impact of secondary emission is small in this case. Further study on the issue is needed in the future.

\section{Distortion versus active power}

The previous section presents the average emission levels of the three turbines over a longer period. During the measurement period, of the order of one week, the wind speed varied a lot in all cases. Unlike the other types of conventional generators, wind turbines show large variations in power production. Variations in power production are associated with variations in the rotation speed on the generator side of converters. The emission is expected to show variations with active power production, among others because of the link between rotational speed and active power production. The relations between the active power production and the current emission have been studied in more details, for the different frequency components.

\subsection{Spectrogram as a function of active power}

In this section a study on the emission as a function of activepower is presented. The harmonic and interharmonic subgroups of the three wind turbines, obtained from the 200-ms windows, are shown in Fig. 5 (Turbine I), Fig. 6 (Turbine II) and Fig. 7 (Turbine III) as a function of the produced power. The upper figure presents the current emission (in ampere) in logarithm (base 10) with colour. The colour scale indicates the magnitude of the harmonic and interharmonic subgroups with red the highest and dark blue the lowest magnitude. The horizontal axis has been obtained by sorting the spectra by the active power, with highest production towards the right. The sorted production values are shown in the bottom curve. The idle states (where the wind turbine is standing still and the converter disconnected, but where a small negative power flows through the turbine transformer due to losses and auxiliary supply) have been removed from the figure. Note that the active-power scale is not linear.

As in Fig. 5, characteristic harmonics (H5 and $\mathrm{H} 13$ ) remain apparent over the whole range of active power production. The low order harmonics, obviously for harmonic 5 , become stronger with the increasing production. The interesting observation is that the emission around harmonic 13 gets stronger at the higher power production, and that the strongest emission shifts to a higher order. The obvious changes occur above 0.5 per unit active-power production (or around data order 850). Another significant observation is that around 0.26 per unit active-power the emission shows a minimum.

For Turbine II (Fig. 6) lower order harmonics increase in magnitude and the maximum emission shifts to higher orders (interharmonics) from around 0.25 per unit active-power production. The emission from harmonic order 10-30 remains present until around 0.5 pu power production. Above around 0.7 pu power blue colour regions between harmonic orders $\mathrm{H} 15-\mathrm{H} 25$ and $\mathrm{H} 30-\mathrm{H} 40$ present low emission. Another observation is that emission at harmonic orders above $\mathrm{H} 35$ increases at $0.2-0.4$ pu power production.

The type- 4 turbine shows another emission pattern, as shown in Fig. 7. The main similarity with the previous two turbines is that low order harmonics, especially interharmonics (e.g. interharmonics 5.5 and 7.5) next to the characteristic harmonics (e.g. harmonics 5 and 7), increase with an increasing power production. The broadband emission from $\mathrm{H} 35$ to $\mathrm{H} 40$ is stronger around rated production. The emission of these orders is relatively low from 0.2 to 0.5 pu active power.

From the above figures, it is concluded that characteristic harmonics are present for any power production; but certain lower order emission increases with production. The increase is mainly in the neighbouring interharmonics of characteristic harmonics. The interharmonic magnitudes exceed those of the characteristic harmonics for certain values of active-power production. Higher order emission presents a number of patterns, which are different for different turbines. 


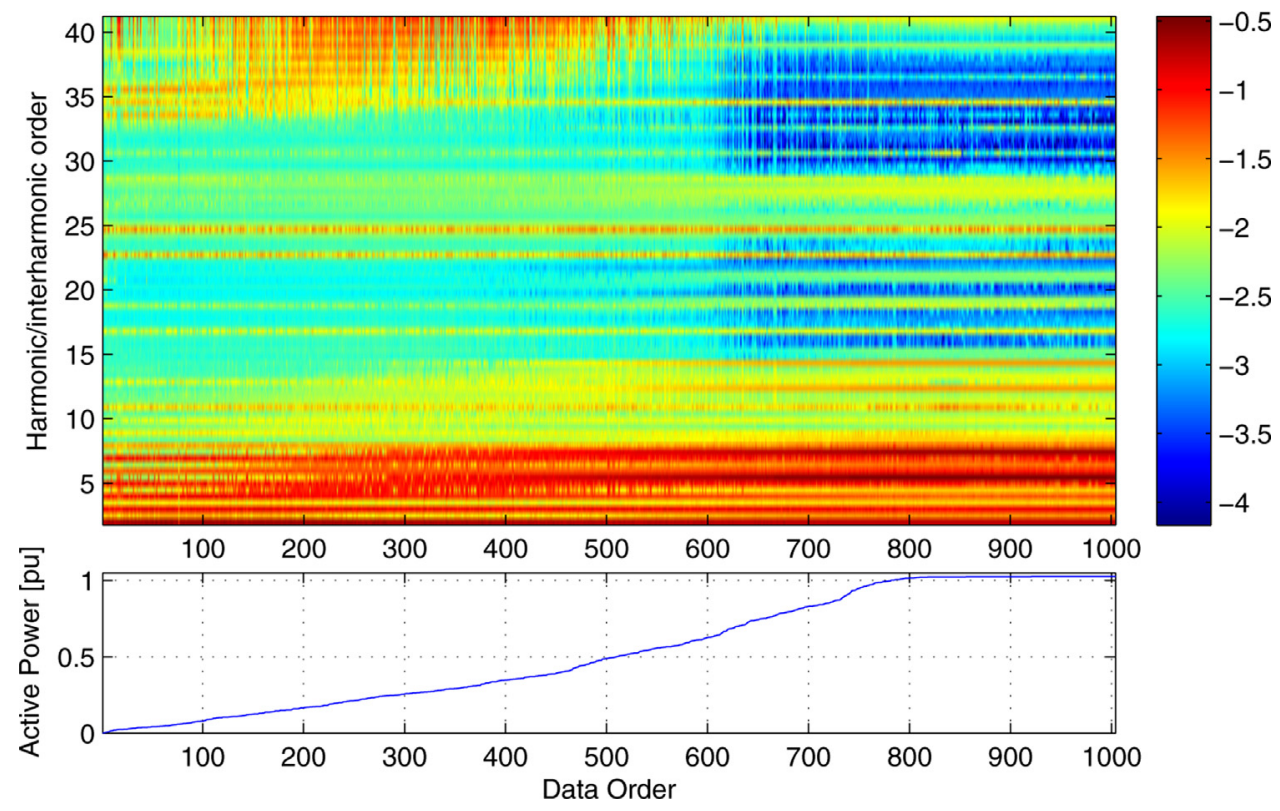

Fig. 6. Turbine II: harmonic (H 2-H 41) and interharmonic (IH 2.5-IH 41.5) subgroups as a function of active power. (For interpretation of the references to colour in the text, the reader is referred to the web version of the article.)
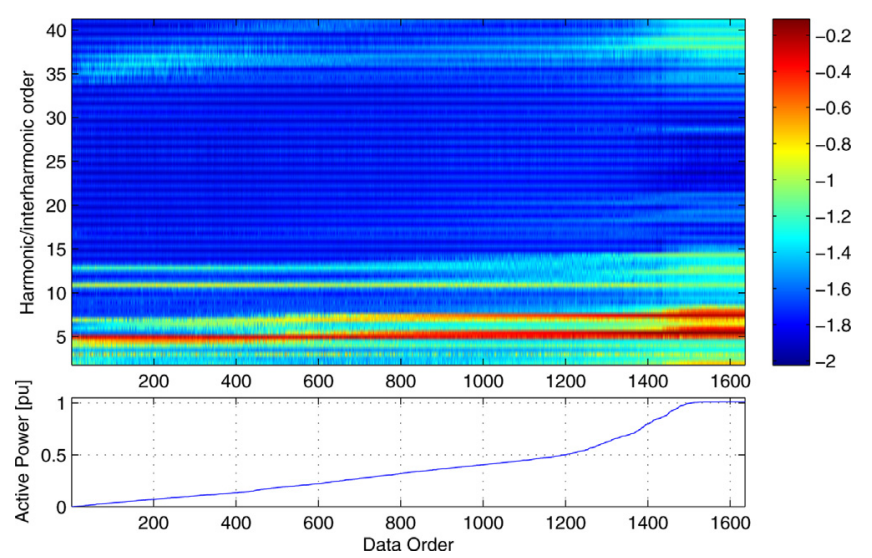

Fig. 7. Turbine III: harmonic ( $\mathrm{H} 2-\mathrm{H} 41)$ and interharmonic (IH 2.5-IH 41.5) subgroups as a function of active power. (For interpretation of the references to colour in the text, the reader is referred to the web version of the article.)

\subsection{THD and TID as a function of active power}

The total harmonic distortions of the three turbines, as a function of active power production, are presented as in Fig. 8. The current THDs were recorded in absolute ampere in Dranetz instrument. The paper [14] presents a constant THC (total harmonic current in percent with the rated wind turbine current), with the increasing active power production. Whereas the paper [15] points out an exponentially decreasing trend (total harmonic distortion as a percentage of fundamental current component) with respect to active power production in a wind farm, and an unapparent trend of that in percent of wind farm rated current. Ref. [15] however shows results for fixed-speed machines, where the mechanism behind the emission is different than for the turbines discussed in this paper.

The total harmonic distortion (THD) versus active power is presented in Fig. 8. The horizontal axis represents the active power in per unit. The total harmonic distortion (THD) (vertical axis) is measured in absolute ampere.

The three wind turbines show a somewhat different relation between emission and the active power production. Turbine I shows an increase, Turbine II a mild decrease and Turbine III remains about the same. Each turbine is different; the measurements together with earlier measurements presented in [14,15] indicate that there is no clear trend for the relation between THD (in ampere) and active-power production. Both Turbine I and Turbine II present a narrow range (by absolute value) of the emission for a certain active power production, if compared to that of Turbine III.

The current total interharmonic distortions (TIDs) are shown in Fig. 9 as a function of active-power production.

All three turbines show a clear increase of TID with active power production. The three trends are a combination of almost linear line with fluctuations at certain active power ranges. There is a drop at 0.26 pu for Turbine I (which is also visible for the THD in Fig. 8, and multiple lines below 0.15 pu for Turbine II and a drop from 0.15 to 0.3 pu for Turbine III.

The TID for a given value of active power production shows less variations than for the THD. There is a strong correlation between the emission and the active power production. The higher active power production, the higher the TID is.

\subsection{Turbine II: individual subgroups as a function of active power}

Next to THD and TID, also the individual harmonic and interharmonic subgroups have been plotted as a function of the active-power production. Some of the results are presented in this and the next section. It is not possible to show all harmonic and interharmonic subgroups, so that certain typical subgroup orders have been chosen to be presented here.

Individual harmonic and interharmonic subgroups show different relations to the active power production. Five subgroup orders have been chosen for Turbine II: 3rd, 5th, 6th, 32nd and 36th; and are shown in Fig. 10. The harmonic subgroups versus active power [pu] are presented on the left, and the interharmonic subgroups are on the right.

The figure shows immediately that there is a wide range in behaviour. There is no general trend for all or most subgroups. In Turbine II, certain harmonic subgroups are not correlated with the active power, especially the lower-order characteristic harmonics, $\mathrm{H} 5, \mathrm{H} 7, \mathrm{H} 11$ and $\mathrm{H} 13$ (similar pattern as $\mathrm{H} 5$ in the figure). The subgroups are within a wide magnitude range as a function of power, 

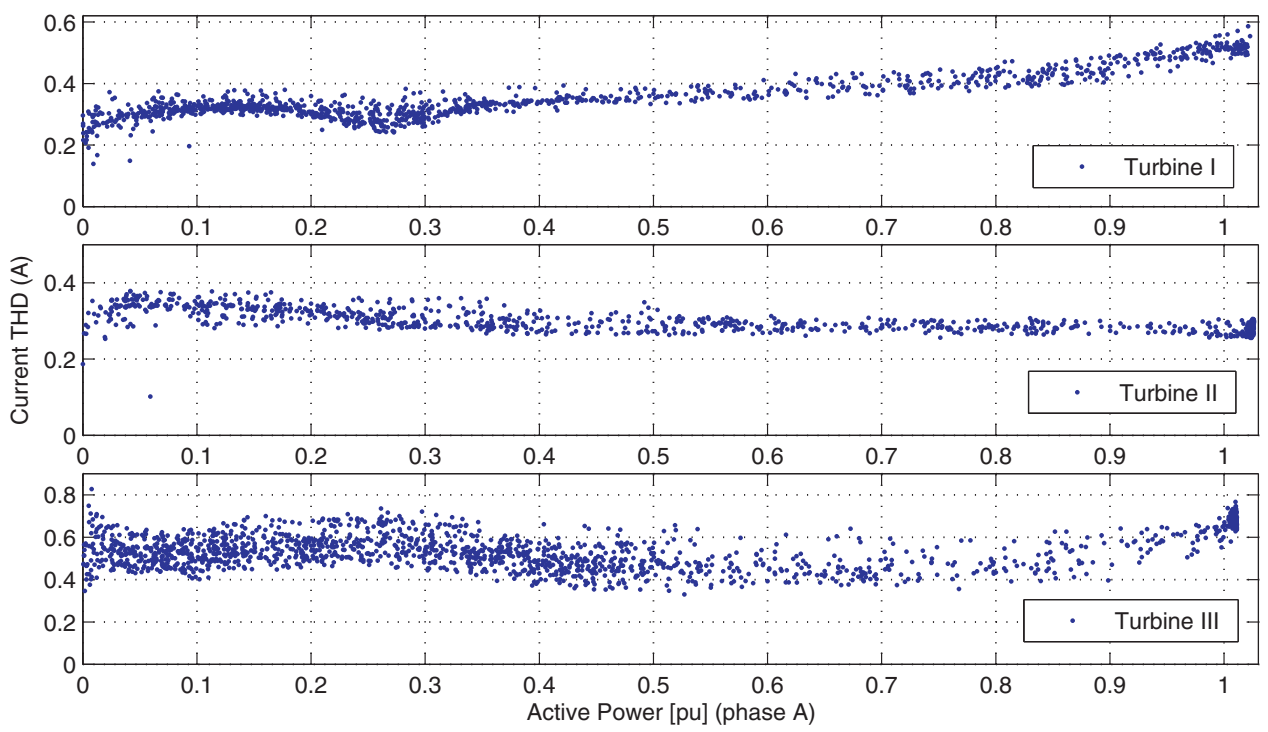

Fig. 8. Total harmonic distortions (THDs) as a function of active power in phase A.

and without an obvious trend with the increasing active power. Other harmonic orders present a narrow magnitude variation as a function of power, e.g. $\mathrm{H} 4, \mathrm{H} 6$ and $\mathrm{H} 8$ (which all show a similar pattern as $\mathrm{H} 6$ in the figure). The higher-order emission decreases with the increasing power production, e.g. H35. Note that the emission level for higher-order components is low compared to the emission for lower-order components.

Interharmonic subgroups present a stronger dependence on the active power than the harmonic subgroups. There are several patterns from the observation: a slightly low emission around $0.3 \mathrm{pu}$ active power (e.g. IH 2 and IH 3); apparent linear and increasing trend associated with several parallel trends below $0.4 \mathrm{pu}$ (interharmonics next to characteristic harmonics as IH5, IH7, etc.); emission with two apparent linear trends associated with emission between the two linear lines as a function of power (e.g. IH 4, IH 6, etc.); and some other irregular patterns such as IH 32 and IH 36.

Although the individual subgroups present different patterns as a function of the active-power production as well as the different emission levels in different power production, there are some similarities between certain orders. Triplen and characteristic harmonics present a low dependence on the active power, whereas other orders, especially interharmonics, present a strong dependence on the active power.

\subsection{Individual subgroups of Turbines I and Turbine III}

The individual subgroups as a function of active power production are presented for the other two wind turbines in Fig. 11. The five chosen individual harmonic orders of each wind turbine present the representative curves for each turbine.

Fig. 11(a) presents typical harmonic and interharmonic subgroups for Turbine I: harmonic and interharmonic orders 3, 7, 12, 16 and 19. The emission at each active power production shows less spread for interharmonic subgroups than for harmonic subgroups. Harmonic subgroup 7, which is similar to orders 5 and 11, shows a large spread for a given active power production. The curve does not show a strong relation between the emission and the active power. There exists an emission drop at around 0.26 pu active power on the increasing trend till 0.8 pu active power, then followed by a decreasing emission.

The results for Turbine III are presented in Fig. 11 (b), with specified orders 3, 4, 6, 7 and 22. Same as the other two turbines, the

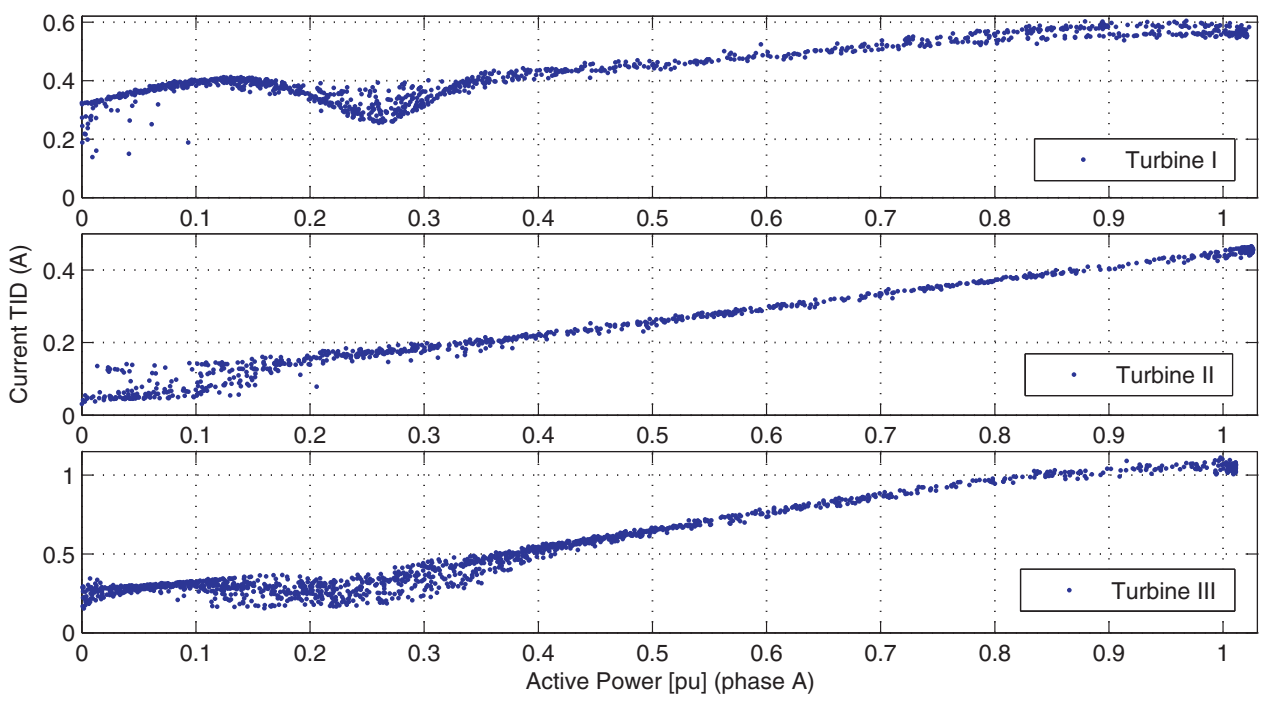

Fig. 9. Total interharmonic distortion (TID) as a function of active power. 


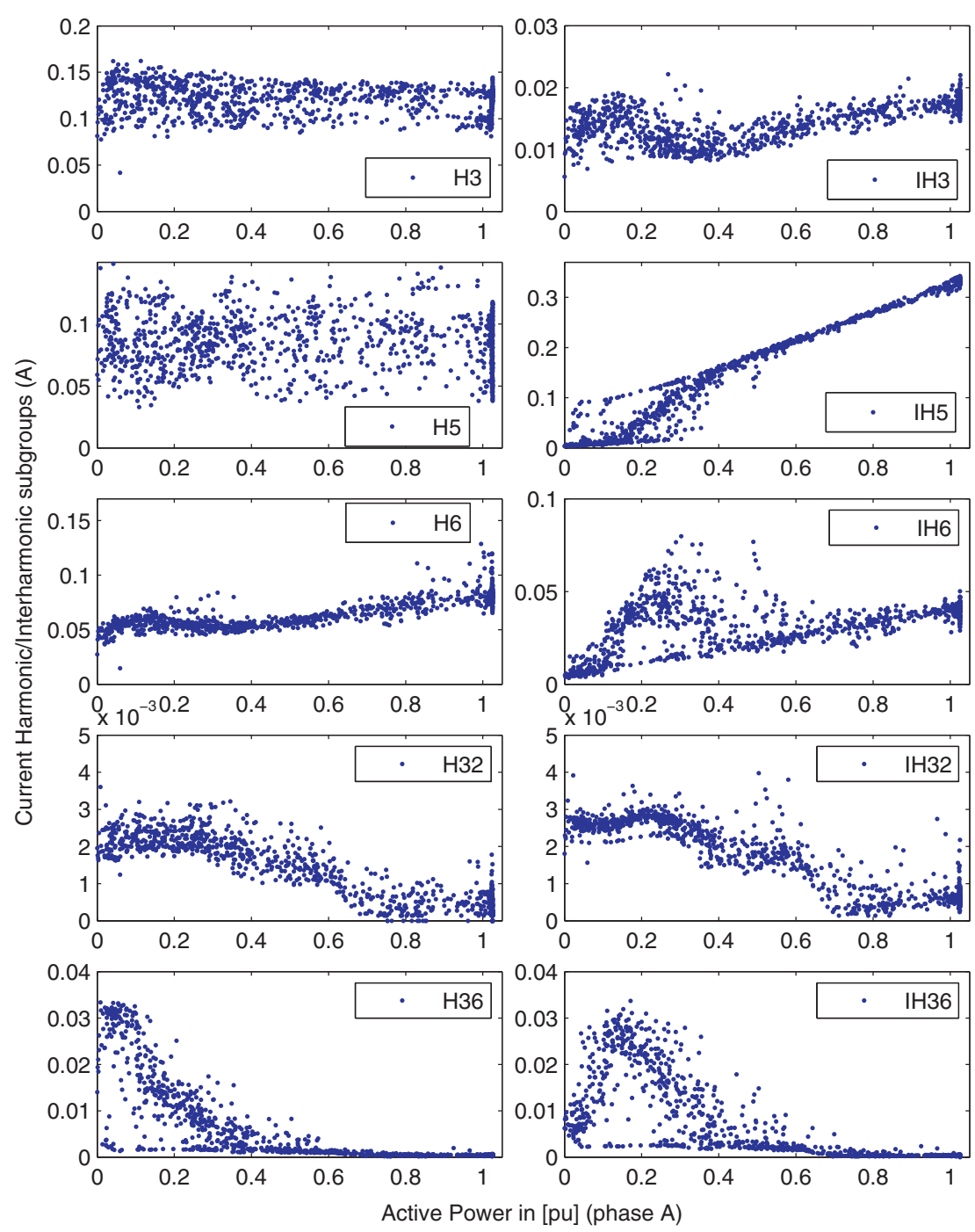

Fig. 10. Turbine II: individual harmonic $(\mathrm{H})$ and interharmonic $(\mathrm{IH})$ subgroups as a function of active power.

low order harmonic subgroups (example as harmonics 3 and 4 presented in the figure) spread in a larger range if compare to the higher orders from the same turbine.

The three turbines present different trends of subgroups, either for the different orders of one turbine or trends between turbines. Individual subgroups of the two turbines present different patterns. Some general patterns can however be observed: the emission for characteristic harmonics is independent on active power; interharmonics (especially neighbouring interharmonics of characteristic harmonic orders) are more dependent on the active power. The spread from the average trend is large for harmonics but small for interharmonics.

\subsection{Quantification study on 5th harmonic and interharmonic}

The standard IEC 61400-21 recommends that the current emission is presented with the power bins: $0,10,20, \ldots, 100 \%$ of active power, where $0,10,20, \ldots, 100 \%$ are the bin midpoints. Examples of patterns for emission versus power production were shown earlier as a scatter plot in Figs. 10 and 11.

The emission for each harmonic and interharmonic subgroup has been quantified per active-power but by its 5 th percentile value, mean value and 95th percentile value. These values, as a function of active power production, are shown in Fig. 12. From the total 1126 measures, there are $190,113,112,109,77,65,68,41,63,34$ and 254 measures from power bin $0 \%$ till power bin $100 \%$.

The 5 th percentile, mean and 95 th percentile values of harmonic subgroup 5 are about the same for the different power bins. The plot also shows a large difference between the 5th and 95th percentiles. It is thus not possible to a give a representative value for the emission within a power bin.

All three values are increasing for interharmonic subgroup 5. Here the observation is a relatively large spread up to $30 \%$ and a small spread for higher power. The relatively large difference between the 5 th percentile and 95th percentile below the $40 \%$ power bin, is also visible in Fig. 10.

This study of this typical pattern shows independence between the harmonic subgroup 5 and the active power, and the strong dependence between the interharmonic subgroup 5 and the active power. It also shows that the spread of the emission values within one power bin is large for the harmonic subgroup but small for the interharmonic subgroup.

\subsection{Quantification of variation per power bin}

The level of variation in emission within the same power bin was shown to be different for different turbines and harmonics in the previous section. It was also shown that the individual 


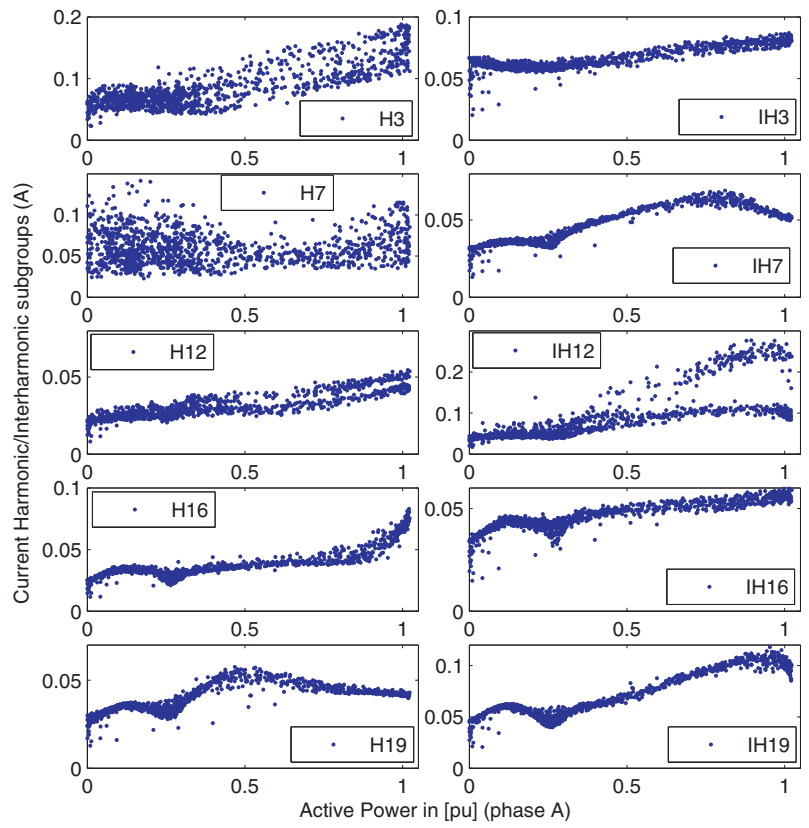

(a)

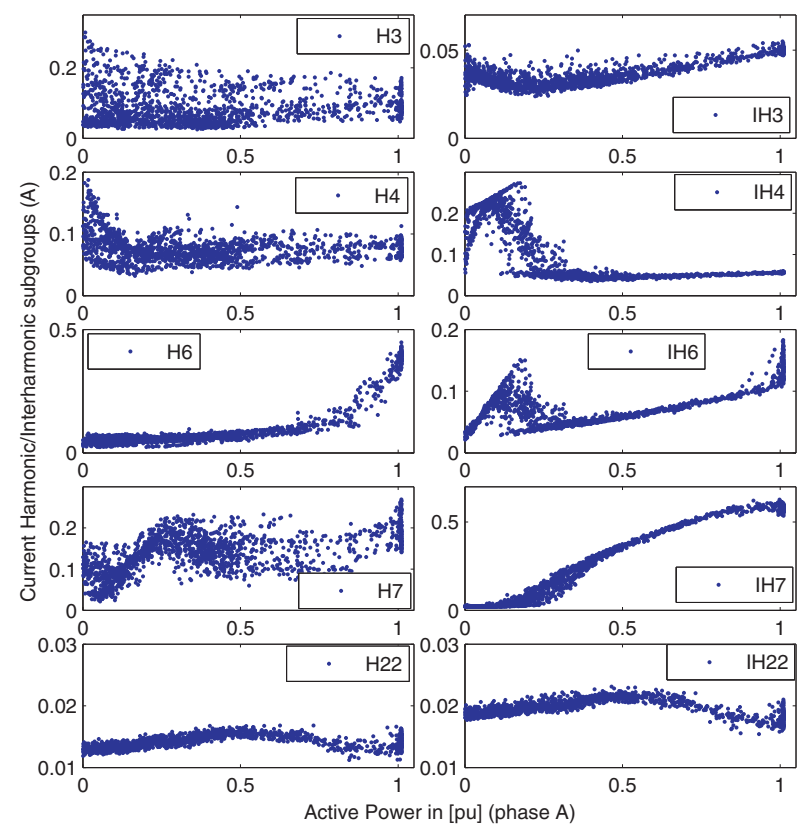

(b)

Fig. 11. Individual harmonic $(\mathrm{H})$ and interharmonic $(\mathrm{IH})$ subgroups as a function of active power in phase A. (a) Turbine I and (b) Turbine III.

subgroups show different trends for emission versus active power. The relation between an individual subgroup and the active-power production is thus different for different subgroups and also for different turbines.

Within a power bin, a larger spread of the emission indicates more independence between the emission and the active power. To quantify the variation of an individual harmonic or interharmonic subgroup within the power bins, the term 'variation index' $K(h)$ at harmonic order $h$ is introduced:

$K(h)=\frac{(1 / 11) \sum_{n=1}^{11}\left(H_{95}(n)-H_{5}(n)\right)}{(1 / 11) \sum_{n=1}^{11} H_{95}(n)}$

where $H_{95}(n)$ and $H_{5}(n)$ are the 95 th percentage and 5 th percentage values at power bin $n ; n$ counts from 1 to 11 and corresponds to 0 , $10 \%, \ldots, 100 \%$ centre points for the active power bins.
A variation index equal to zero occurs when the 5th and 95th percentiles are equal to each other for all 11 power bins. The variation index is equal to unity when all 5 percentile values are equal to zero. In that case the relative variation is very big. The lower the variation index, the more the emission only depends on the active-power production.

Fig. 13 presents the variation index for the three turbines, separately for harmonic and interharmonic subgroups.

With Turbines I and III the variation index is much lower for interharmonics than for harmonics up to order 15 (Fig. 13). For higher order the variation index is slightly lower for interharmonics, but the difference is small. For Turbine III the latter is the case also for lower order, as shown in Table 2. Higher order interharmonics for Turbine II show a high variation index, close to unity. For Turbine I and Turbine III the variation index decreases with increasing harmonic order. For Turbine II, large variation

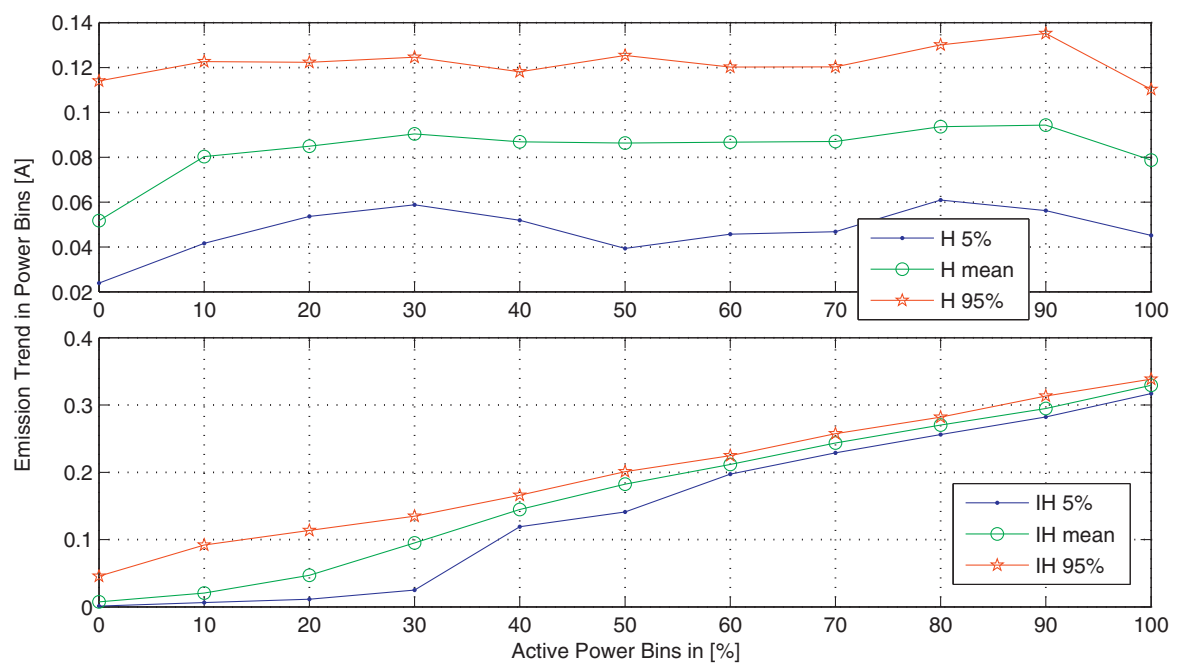

Fig. 12. Turbine II: $5 \%$ (5th percentile value), mean and $95 \%$ (95th percentile value) of harmonic (H) 5 and interharmonic (IH) 5 subgroups as a function of active power bin according to requirement in IEC 61400-21. 

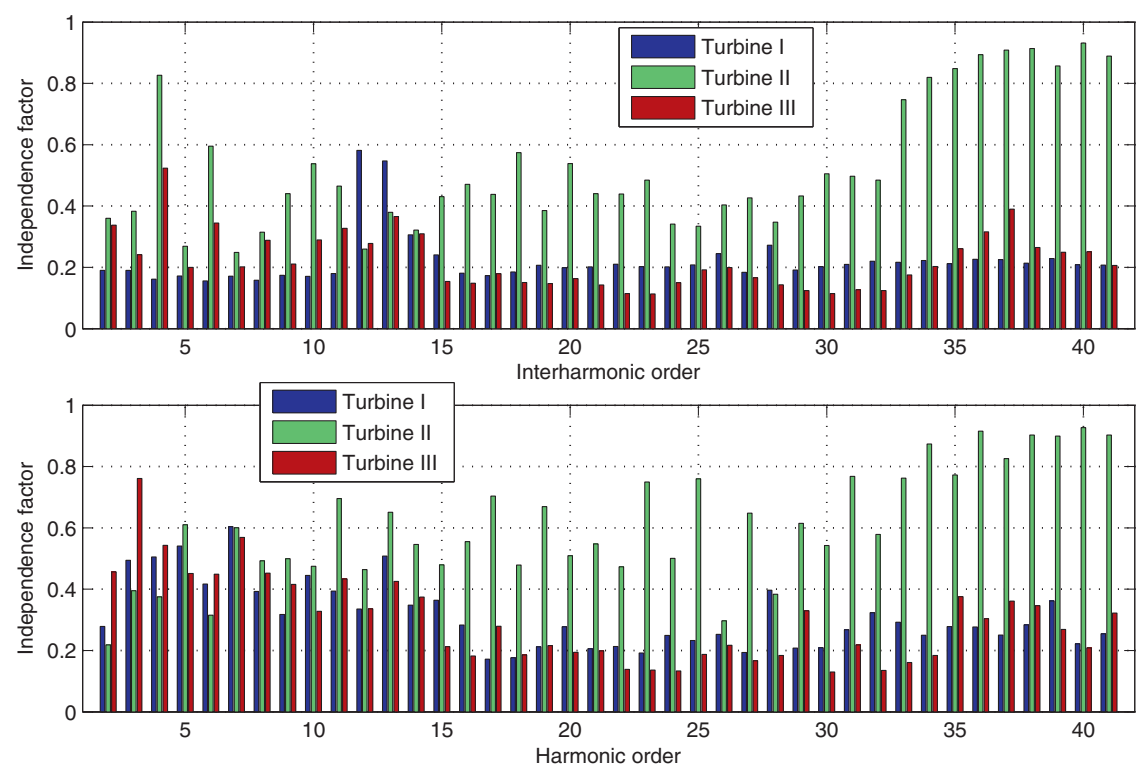

Fig. 13. Interharmonic (upper) and harmonic (lower) variation index.

Table 2

Average variation index over 10 orders, for the three turbines.

\begin{tabular}{llllllll}
\hline & H2-10 & IH 2-10 & H 11-20 & IH 11-20 & H 21-30 & IH 21-30 & H 31-41 \\
\hline T I & 0.4439 & 0.1714 & 0.3071 & 0.2800 & 0.2352 & 0.2118 \\
T II & 0.4423 & 0.4417 & 0.5751 & 0.4263 & 0.5516 & 0.4155 \\
T III & 0.4918 & 0.2930 & 0.2839 & 0.2223 & 0.1821 & 0.2782 \\
\hline
\end{tabular}

index is observed above order 15 , for harmonics as well as for interharmonics.

\section{Conclusion}

The three turbines show different spectra, but some general observations can be made. The spectrum is for all three turbines a combination of narrowband and broadband components. The consequence of this is that next to integer harmonics, the spectra have large interharmonic contents. The levels of characteristic harmonics are relatively low, where the emission limits in IEEE Std.519 have been used as a reference. Interharmonics and even harmonics are relatively high, especially for higher orders. The rule that emission is dominated by odd non-triplen harmonics does not hold for wind turbines.

Contrary to earlier publications, the measurements showed different relations between THD and active-power production for different turbines. The variation of THD with active power is relatively small. The relation between total interharmonic distortion (TID) and active-power production is similar for the three turbines; an increase in TID with increasing active-power production. Individual harmonic and interharmonic subgroups show different relations between emission and active-power production. Characteristic harmonics are shown to be independent on the active power. They show large variations even for small variations in active power. Whereas the neighbouring interharmonics present a strong dependency on the active power. A general observation is that the dominant interharmonics increase with active power production, whereas the dominant harmonics remain more constant. TID and individual harmonics have not been studied in other publications.

The spectrogram sorted by active-power production has shown to be a suitable graphical method for illustrating the changes in emission spectrum with active power production. For the three turbines studied in this paper, this type of spectrogram showed that the character of the spectrum changes with active-power production, especially where it concerns interharmonics.

The emission of a wind turbine is not only determined by the active power production. For a given amount of active power production, there is a range in measured values of the emission. This range is small for part of the harmonic and interharmonic subgroups and large for others. There is no obvious pattern observable in this and the range is different for different frequency components and for different turbines.

To know the emission from a wind turbine, it is thus not sufficient to know the active-power production. This also means that the reporting method according to IEC 61400-21 has its limits. This method only reports the maximum emission for each power bin, not the spread of the emission. For some applications this may be sufficient, but for research purposes, to understand the emission or for serious planning efforts more information is needed.

Further work needed includes a study of multiple trends of emission as a function of active-power at certain orders (e.g. interharmonic 5 together with interharmonic 7) and a study of the shift towards higher orders for increasing active-power production. Further work is also needed towards a method for distinguishing between emission driven by the power electronics in the turbine and emission driven by the background voltage distortion. An evaluation of the impact of even and interharmonics on the grid is needed, including a possible reevaluation of limits for these frequency components.

\section{References}

[1] International Electrotechnical Commission, Grid integration of large-capacity Renewable Energy sources and use of large-capacity Electrical Energy Storage, IEC White Paper, October 2012. 
[2] F. Blaabjerg, M. Liserre, K. Ma, Power electronics converters for wind turbine systems, IEEE Transactions on Industry Applications 48 (2) (2012) 708-719, http://dx.doi.org/10.1109/TIA.2011.2181290.

[3] J. Arrillaga, N.R. Watson, Power System Harmonics, 2nd edition, Wiley, London, U.K., 2003.

[4] N. Mohan, T. Undeland, W. Robbins, Power Electronics: Converters, Applications, and Design, vol. 1, John Wiley \& Sons, New York, 2003.

[5] S. Heier, Grid Integration of Wind Energy Conversion Systems, 2nd edition, John Wiley \& Sons, Ltd., Chichester, England, 2006.

[6] R. Teodorescu, M. Liserre, P. Rodriguez, Grid Converters for Photovoltaic and Wind Power Systems, 1st edition, John Wiley \& Sons, Ltd., New York, 2011.

[7] B. Wu, High-Power Converters and AC Drives, 1st edition, Wiley-IEEE Press, Piscataway, NJ, 2006

[8] B. Wu, Y. Lang, N. Zargari, S. Kouro, Power Conversion and Control of Wind Energy Systems, IEEE Power Engineering Series, Wiley, Hoboken, NJ, 2011.

[9] R. Dugan, M. McGranaghan, S. Santosa, H. Beaty, Electric Power Systems Quality, 3rd edition, McGraw-Hill, New York, 2012.

[10] M. Bollen, I. Gu, Signal Processing of Power Quality Disturbances, IEEE Press Series on Power Engineering, Wiley, New York, 2006.

[11] A. Baggini, Z. Hanzelkar, Voltage and current harmonics, in: Handbook of Power Quality, A. Baggini edition, John Wiley \& Sons, Ltd., Chichester, UK, 2008.

[12] International Electrotechnical Commission, IEC 61400-21, 2008.

13] CENELEC, IEC 61000-4-7, Electromagnetic compatibility (EMC) - Part 4-7: Testing and measurement techniques - general guide on harmonics and interharmonics measurements and instrumentation, for power supply systems and equipment connected thereto, IEC 61000-4-7, 2002.
[14] S.T. Tentzerakis, S.A. Papathanassiou, An investigation of the harmonic emissions of wind turbines, IEEE Transactions on Energy Conversion 22 (1) (2007) 150-158, http://dx.doi.org/10.1109/TEC.2006.889607.

[15] L. Sainz, J. Mesas, R. Teodorescu, P. Rodriguez, Deterministic and stochastic study of wind farm harmonic currents, IEEE Transactions on Energy Conversion 25 (4) (2010) 1071-1080, http://dx.doi.org/10.1109/TEC.2010.2045379.

[16] H. Emanuel, M. Schellschmidt, S. Wachtel, S. Adloff, Power quality measurements of wind energy converters with full-scale converter according to IEC 61400-21, in: 10th International Conference on Electrical Power Quality and Utilisation (EPQU), 2009, pp. 1-7, http://dx.doi.org/10.1109/ EPQU.2009.5318814.

[17] J. Arrillaga, N. Watson, S. Chen, Power System Quality Assessment, 1st edition, John Wiley \& Sons, New York, 1999.

[18] J. Meyer, P. Schegner, K. Heidenreich, Harmonic summation effects of modern lamp technologies and small electronic household equipment, in: Proceedings of the 21st International Conference and Exhibition on Electricity Distribution, Frankfurt, Germany, 2011.

[19] Y. Chen, M. Bollen, M. Martins, Application of transfer function based harmonic study method to an offshore wind farm, in: Int. Workshop on Wind Power Integration, 2012

[20] S. Rönnberg, A. Larsson, M. Bollen, J. Schanen, A simple model for interaction between equipment at a frequency of some tens of $\mathrm{kHz}$, in: Proceedings of the 21st International Conference and Exhibition on Electricity Distribution, Frankfurt, Germany, 2011. 